\title{
Preference Relation-based Markov Random Fields for Recommender Systems
}

\author{
Shaowu Liu'1,2 Gang $\mathrm{Li}^{1}$. Truyen $\operatorname{Tran}^{3}$. \\ Yuan Jiang 4
}

Received: 15 February 2016/ Accepted: 24 October 2016 / Published online: 14 November 2016

(C) The Author(s) 2016

\begin{abstract}
A preference relation-based Top- $\mathrm{N}$ recommendation approach is proposed to capture both second-order and higher-order interactions among users and items. Traditionally Top- $\mathrm{N}$ recommendation was achieved by predicting the item ratings first, and then inferring the item rankings, based on the assumption of availability of explicit feedback such as ratings, and the assumption that optimizing the ratings is equivalent to optimizing the item rankings. Nevertheless, both assumptions are not always true in real world applications. The proposed approach drops these assumptions by exploiting preference relations, a more practical user feedback. Furthermore, the proposed approach enjoys the representational power of Markov Random Fields thus side information such as item and user attributes can be easily incorporated. Comparing to related work, the proposed approach has the unique property of modeling both second-order and higher-order interactions among users and items. To the best of our knowledge, this is the first time both types of interactions have been captured in preference-relation based methods. Experimental results on public datasets demonstrate that
\end{abstract}

Editors: Geoff Holmes, Tie-Yan Liu, Hang Li, Irwin King and Zhi-Hua Zhou.

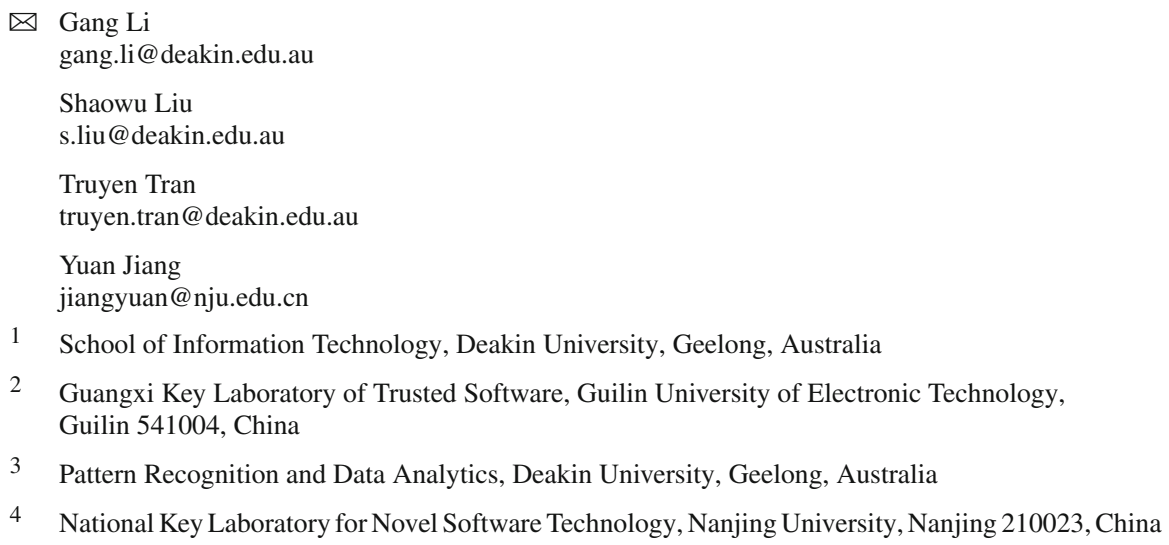

4 National Key Laboratory for Novel Software Technology, Nanjing University, Nanjing 210023, China 
both types of interactions have been properly captured, and significantly improved Top-N recommendation performance has been achieved.

Keywords Recommender systems · Collaborative filtering · Preference relation · Pairwise preference $\cdot$ Markov Random Fields

\section{Introduction}

Recommender Systems (RecSys) aim to recommend users with some of their potentially interesting items, which can be virtually anything ranging from movies to tourism attractions. To identify the appropriate items, RecSys attempts to exploit user preferences (Koren et al. 2009) and various side information including content (Balabanović and Shoham 1997; Basilico and Hofmann 2004), temporal dynamics (Koren 2010), and social relations (Ma et al. 2011). By far, Collaborative Filtering (Koren et al. 2009) is one of the most popular RecSys techniques, which exploits user preferences, especially in the form of explicit absolute ratings. Nevertheless, relying solely on absolute ratings is prone to the cold-start problem (Schein et al. 2002) where few ratings are known for cold users or items. To alleviate the cold-start problem, additional information, which is usually heterogeneous (Basilico and Hofmann 2004) and implicit (Rendle et al. 2009) must be acquired and exploited.

Recently, a considerable literature (Liu et al. 2009; Rendle et al. 2009; Desarkar et al. 2012; Brun et al. 2010; Shi et al. 2010) has grown up around the theme of relative preferences. The underlying motivation is that relative preferences are often easier to collect and more reliable as a measure of user preferences. For example, it can be easier for users to tell which item is preferable than expressing the precise degree of liking. Furthermore, studies (Koren and Sill 2011; Brun et al. 2010) have reported that absolute ratings may not be completely trustworthy. For example, rating 4 out of 5 may in general indicate high quality, but it can mean just average for critics. In fact, users' quantitative judgment can be affected by irrelevant factors such as their mood when rating, and this is called misattribution of memory (Schacter and Dodson 2001).

While users are not good at making consistent quantitative judgments, the preference relation ( $\mathrm{PR}$ ), as a kind of relative preference, has been considered as more consistent across like-minded users (Brun et al. 2010; Desarkar et al. 2010, 2012). By measuring the relative order between items, the $P R$ is usually less variant to irrelevant factors. For example, a user in a bad mood may give lower ratings to all items but the relative orderings between items remain the same. Being a more reliable type of user preference, $P R$ is also easier to collect comparing to ratings as it can be inferred from implicit feedback. For example, the $P R$ between two items can be inferred by comparing their ratings, page views, played counts, mouse clicks, etc. This property is important as not all users are willing to rate their preferences, where collecting feedback implicitly delivers a more user-friendly recommender system. In addition, the output of RecSys is often the item ranking, which is also a type of relative preference, and therefore relative preference is more natural input than absolute ratings (Tran et al. 2012; Koren and Sill 2011).

While $P R$ captures user preferences in pairwise form, most existing works (Koren and Sill 2011; Liu et al. 2014) take the pointwise approach to exploiting ordinal properties possessed by absolute ratings. To accept the $P R$ as input and output item rankings, pairwise approaches to RecSys have recently emerged in two forms: memory-based (Brun et al. 2010) and modelbased (Liu et al. 2009; Rendle et al. 2009; Desarkar et al. 2012). These studies have shown the feasibility of $P R$-based methods, and demonstrated competitive performance comparing 
to their underlying models, such as memory-based K-Nearest Neighbor (KNN) (Brun et al. 2010) and model-based Matrix Factorization (MF) (Desarkar et al. 2012).

However, the limitations of these underlying models have constrained the potential of their $P R$ extensions. More specifically, both $K N N$ and $M F$ based methods can only capture one type of information at a time, while both the local and the global information are essential in achieving good performance (Tran et al. 2009; Koren 2008; Liu et al. 2014). We refer to these two types of information as the local and the global structures of the preferences:

Local Structure The local structure (LS) refers to the second-order interactions between similar users or items. This type of information is often used by neighborhood-based collaborative filtering, in which the predictions are made by looking at the neighborhood of users (Resnick et al. 1994) or items (Sarwar et al. 2001). LS-based approaches ignore the majority of preferences in making predictions, but are effective when the user or item correlations are highly localized.

Global Structure The global structure (GS) refers to the weaker but higher-order interactions among all users and items. This type of information is often used by latent factor models such as Matrix Factorization (Koren et al. 2009), which aim to discover the latent factor space in the preferences. $G S$-based approaches are often competitive in terms of accuracy and computational efficiency (Koren et al. 2009).

Previous studies have suggested that these two structures are complementary since they address different aspects of the preferences (Tran et al. 2009; Koren 2008; Liu et al. 2014). However, to the best of our knowledge, there is yet no $P R$-based method that can capture both $L S$ and $G S$. Another problem of existing $P R$-based methods is that side information such as item content and user attributes can't be easily incorporated, which is critical in coldstart cases. All the above reasoning lead to a desired model with the following properties: (1) Accept $P R$ as input; (2) Capture both $L S$ and $G S$; (3) Side information can be easily incorporated; (4) Output item rankings.

Recent advances in Markov Random Fields-based RecSys (Tran et al. 2009; Defazio and Caetano 2012; Liu et al. 2014) have made it possible to achieve the above objectives. MRFbased RecSys was first developed in Tran et al. (2009) to capture both $L S$ and GS. Later on, it has been extended in Liu et al. (2014) to exploit ordinal properties possessed by absolute ratings. Nevertheless, all of these attempts rely on absolute ratings.

This paper aims to push the $M R F$-based RecSys one step further by fitting it into the $P R$ framework, namely Preference Relation-based Markov Random Fields (PrefMRF) and Preference Relation-based Conditional Random Fields (PrefCRF) when side information is incorporated. The remaining parts of this paper are organized as follows. Section 2 introduces the concepts of $P R$-based RecSys and formalizes the problem, followed by a review of related work. Section 3 is devoted to the proposed PrefMRF and PrefCRF models. Benchmark results on Top-N recommendation are presented in Sect. 4. Finally, Sect. 5 concludes this paper by summarizing the main contributions and envisaging future work.

\section{Preliminaries and related work}

Recommender Systems (RecSys) aim at predicting users' future interest in items, and the recommendation task can be considered as a preference learning problem, which aims to construct a predictive preference model from observed preference information (Mohri et al. 2012). Existing preference learning methods are based on different learning to rank approaches (Fürnkranz and Hüllermeier 2010). Among them, the pointwise approach is the 
choice of most RecSys (Sarwar et al. 2001; Koren 2008), which exploit absolute ratings, though the pairwise approach that exploits $P R$ has been largely overlooked until recently. The rest of this section describes the basic concepts and formalizes the $P R$-based RecSys followed by a review of related work.

\subsection{Preference relation}

Preference relation has been widely studied in the field of Information Retrieval (Cohen et al. 1999; Fürnkranz and Hüllermeier 2003; Freund et al. 2003; Jin et al. 2002). Nevertheless, PR-based RecSys have only emerged recently (Liu et al. 2009; Rendle et al. 2009; Brun et al. 2010; Desarkar et al. 2012). A preference relation (PR) encodes user preferences in the form of pairwise ordering between items. This representation is a useful alternative to absolute ratings for three reasons.

Firstly, $P R$ is more consistent across like-minded users (Brun et al. 2010; Desarkar et al. 2012 ) as it is invariant to many irrelevant factors, such as mood. Secondly, $P R$ is a more natural and direct input for Top-N recommendation, as both the input and the output are relative preferences. Finally, and perhaps most importantly, $P R$ can be inferred from implicit feedback such as page views and stayed time. This property provides an opportunity to utilize the vast amount of implicit data that has already been collected over the years, e.g., activity $\log s$. With these potential benefits, we shall take a closer look at the $P R$, and investigate how they can be utilized in RecSys.

We formally define the $P R$ as follows. Let $\mathcal{U}=\{u\}^{n}$ and $\mathcal{I}=\{i\}^{m}$ denote the set of $n$ users and $m$ items, respectively. The preference of a user $u \in \mathcal{U}$ between items $i$ and $j$ is encoded as $\pi_{u i j}$, which indicates the strength of user $u$ 's $P R$ for the ordered item pair $(i, j)$. A higher value of $\pi_{u i j}$ indicates a stronger preference on the first item over the second item.

Definition 1 (Preference Relation) The preference relation is defined as

$$
\pi_{u i j}=\left\{\begin{array}{l}
\left(\frac{2}{3}, 1\right] \text { if } i \succ j(u \text { prefers } i \text { over } j) \\
{\left[\frac{1}{3}, \frac{2}{3}\right] \text { if } i \simeq j(i \text { and } j \text { are equally preferable to } u)} \\
{\left[0, \frac{1}{3}\right) \text { if } i \prec j(u \text { prefers } j \text { over } i)}
\end{array}\right.
$$

where $\pi_{u i j} \in[0,1]$ and $\pi_{u i j}=1-\pi_{u j i}$.

This definition is similar to Desarkar et al. (2012), however, we allocate an interval for each preference category, i.e., preferred, equally preferred, and less preferred. Indeed, each preference category can be further broken down into more intervals.

Similar to Brun et al. (2010), the $P R$ can be converted into user-wise preferences over items.

Definition 2 (User-wise Preference) The user-wise preference is defined as

$$
p_{u i}=\frac{\sum_{j \in \mathcal{I}_{u}} \llbracket \pi_{u i j}>\frac{2}{3} \rrbracket-\sum_{j \in \mathcal{I}_{u}} \llbracket \pi_{u i j}<\frac{1}{3} \rrbracket}{\left|\Pi_{u i}\right|}
$$

where $\llbracket \cdot \rrbracket$ gives 1 for true and 0 for false, $\mathcal{I}_{u}$ is the set of items related to user $u$, and $\Pi_{u i}$ is the set of user $u$ 's PR related to item $i$.

The user-wise preference $p_{u i}$ falls in the interval $[-1,1]$, where -1 and 1 indicate that item $i$ is the least or the most preferred item for user $u$, respectively. The user-wise preference measures the relative position of an item for a particular user, which is different from absolute ratings. 


\subsection{Problem statement}

Generally, the task of $P R$-based RecSys is to take $P R$ as input and output Top-N recommendations. Specifically, let $\pi_{u i j} \in \Pi$ encode the $P R$ of each user $u \in \mathcal{U}$. Each $\pi_{u i j}$ is defined over an ordered item pair $(i, j)$, denoting $i \prec j, i \simeq j$, or $i \succ j$ as described in Eq. (2.1). The goal is to estimate the value of each unknown $\pi_{u i j} \in \Pi_{\text {unknown }}$, such that $\hat{\pi}_{u i j}$ approximates $\pi_{u i j}$. This can be considered as an optimization task performed directly on the $P R$ :

$$
\min _{\hat{\Pi}} \sum_{\hat{\pi}_{u i j} \in \hat{\Pi}}\left(\pi_{u i j}-\hat{\pi}_{u i j}\right)^{2}
$$

However, it can be easier to estimate the $\hat{\pi}_{u i j}$ by the difference between the two userwise preferences $p_{u i}$ and $p_{u j}$, i.e., $\hat{\pi}_{u i j}=\phi\left(\hat{p}_{u i}-\hat{p}_{u j}\right)$, where $\phi(\cdot)$ is a function that bounds the value into $[0,1]$ and ensures $\phi(0)=0.5$. For example, the inverse-logit function $\phi(x)=\frac{e^{x}}{1+e^{x}}$ can be used when user-wise preferences involve large values. Therefore, the objective of this paper is to solve the following optimization problem:

$$
\min _{\hat{\mathbf{p}}_{u}} \sum_{\left(\hat{p}_{u i}, \hat{p}_{u j}\right) \in \hat{\mathbf{p}}_{u}}\left(\pi_{u i j}-\phi\left(\hat{p}_{u i}-\hat{p}_{u j}\right)\right)^{2}
$$

which optimizes the user-wise preferences directly, and Top-N recommendations can be obtained by simply sorting the estimated user-wise preferences.

\subsection{Related work}

User preferences can be modeled in three types: pointwise, pairwise, and listwise. Though RecSys is not limited to the pointwise absolute ratings, the recommendation task is usually considered as a rating prediction problem. Recently, a considerable literature (Liu et al. 2009; Rendle et al. 2009; Desarkar et al. 2012; Brun et al. 2010; Shi et al. 2010; Gantner et al. 2010) has grown up around the theme of relative preferences, especially the pairwise $P R$. Meanwhile, the recommendation task is also shifting from rating prediction to item ranking (Weimer et al. 2007; Shi et al. 2010; McNee et al. 2006), in which the ranking itself is also of relative preferences.

The use of relative preferences has been widely studied in the field of Information Retrieval for learning to rank tasks. Recently, PR-based (Liu et al. 2009; Rendle et al. 2009; Desarkar et al. 2012; Brun et al. 2010) and listwise-based (Shi et al. 2010) RecSys have been proposed. Among them, the $P R$-based approach is the most popular, which can be further categorized as memory-based methods (Brun et al. 2010) that capture local structure and model-based methods (Liu et al. 2009; Rendle et al. 2009; Desarkar et al. 2012) that capture global structure. To the best of our knowledge, there is yet no $P R$-based method that can capture both $L S$ and $G S$.

Advances in Markov Random Fields (MRF) and its extension Conditional Random Fields (CRF) have made it possible to utilize both $L S$ and $G S$ by taking advantages of MRF's powerful representation capability. Nevertheless, exploiting the $P R$ is not an easy task for $M R F$ and $C R F$ (Tran et al. 2009; Liu et al. 2014). This observation leads to a natural extension of unifying the MRF models with the $P R$-based models, to complement their strengths. We summarize the capabilities of the existing and our proposed PrefMRF and PrefCRF models in Table 1. 
Table 1 Capabilities of different methods

\begin{tabular}{|c|c|c|c|c|c|}
\hline Method & Input & Output & LS & GS & Side information \\
\hline Pointwise Memory-based & Ratings & Ratings & $\checkmark$ & & \\
\hline Pointwise Model-based & Ratings & Ratings & & $\checkmark$ & \\
\hline Pointwise Hybrid & Ratings & Ratings & $\checkmark$ & $\checkmark$ & \\
\hline Pairwise Memory-based & Preference relations & Item rankings & $\checkmark$ & & \\
\hline Pairwise Model-based & Preference relations & Item rankings & & $\checkmark$ & \\
\hline PrefMRF & Preference relations & Item rankings & $\checkmark$ & $\checkmark$ & \\
\hline PrefCRF & Preference relations & Item rankings & $\checkmark$ & $\checkmark$ & $\checkmark$ \\
\hline
\end{tabular}

Best results are given in bold

Table 2 Summary of major notations

\begin{tabular}{ll}
\hline Notations & Mathematical meanings \\
\hline $\mathcal{U}$ & The set of users \\
$\mathcal{I}$ & The set of items \\
$\Pi$ & The set of preference relations \\
$p_{u i}$ & The user-wise preference of user $u$ on item $i$ \\
$\mathcal{G}$ & An undirected graph encodes \\
$\mathcal{V}$ & relations of user-wise preferences \\
$\mathcal{E}$ & The set of vertices each represents a user-wise \\
$f_{u v}$ & preference \\
$f_{i j}$ & The set of edges each connects two vertices \\
$w_{u v}$ & The correlation feature between users $u$ and $v$ \\
$w_{i j}$ & The correlation feature between items $i$ and $j$ \\
$Q\left(p_{u i} \mid u, i\right)$ & The weight associated to the user-user correlation \\
$\mathbf{0}$ & feature $f_{u v}$ \\
& The weight associated to the item-item correlation \\
& feature $f_{i j}$ \\
The ordinal distribution produced by PrefNMF & The side information, e.g., user attributes and item \\
& content
\end{tabular}

\section{Methodology}

In this section, we propose Preference Relation-based Markov Random Fields (PrefMRF) to model the $P R$ and capture both $L S$ and GS. When side information is taken into consideration, this model extends to Preference Relation-based Conditional Random Fields (PrefCRF). In this work, we exploit $L S$ in terms of the item-item correlations as well as the user-user correlations. The rest of this section introduces the concept of the Preference Relationbased Matrix Factorization (PrefNMF) (Desarkar et al. 2012) that will be our underlying model, followed a detailed discussion of the PrefMRF and PrefCRF on issues including feature design, parameter estimation, and predictions. For ease of reference, notations used throughout this paper are summarized in Table 2. The letters $u, v, a, b$ represent users, and the letters $i, j, k, l$ represent items. 


\subsection{Preference Relation-based Matrix Factorization}

Matrix Factorization (MF) (Koren et al. 2009) is a popular approach to RecSys that has mainly been applied to absolute ratings. Recently, the PrefNMF (Desarkar et al. 2012) model was proposed to adopt $P R$ input for $M F$ models. Like traditional $M F$ models, the PrefNMF model discovers the latent factor space shared between users and items, where the latent factors describe both the taste of users and the characteristics of items. The attractiveness of an item to a user is then measured by the inner product of their latent feature vectors.

\subsubsection{Point estimation}

The PrefNMF model described in Desarkar et al. (2012) provides a point estimation to each preference, i.e., a single value. Formally, each user $u$ is associated with a latent feature vector $\mathbf{u}_{u} \in \mathbb{R}^{k}$ and each item $i$ is associated with a latent feature vector $\mathbf{v}_{i} \in \mathbb{R}^{k}$, where $k$ is the dimension of the latent factor space. The attractiveness of items $i$ and $j$ to the user $u$ are $\mathbf{u}_{u}^{\top} \mathbf{v}_{i}$ and $\mathbf{u}_{u}^{\top} \mathbf{v}_{j}$, respectively. When $\mathbf{u}_{u}^{\top} \mathbf{v}_{i}>\mathbf{u}_{u}^{\top} \mathbf{v}_{j}$ the item $i$ is said to be more preferable to the user $u$ than the item $j$, i.e., $i \succ j$. The strength of this preference relation $\pi_{u i j}$ can be estimated by $\mathbf{u}_{u}^{\top}\left(\mathbf{v}_{i}-\mathbf{v}_{j}\right)$, and the inverse-logit function is applied to ensure $\hat{\pi}_{u i j} \in[0,1]$ :

$$
\hat{\pi}_{u i j}=\frac{e^{\mathbf{u}_{u}^{\top}\left(\mathbf{v}_{i}-\mathbf{v}_{j}\right)}}{1+e^{\mathbf{u}_{u}^{\top}\left(\mathbf{v}_{i}-\mathbf{v}_{j}\right)}}
$$

The latent feature vectors $\mathbf{u}_{u}$ and $\mathbf{v}_{i}$ are learned by minimizing regularized squared error with respect to the set of all known preference relations $\Pi$ :

$$
\min _{\mathbf{u}_{u}, \mathbf{v}_{i} \in \mathbb{R}^{k}} \sum_{\pi_{u i j} \in \Pi \wedge(i<j)}\left(\pi_{u i j}-\hat{\pi}_{u i j}\right)^{2}+\lambda\left(\left\|\mathbf{u}_{u}\right\|^{2}+\left\|\mathbf{v}_{i}\right\|^{2}\right)
$$

where $\lambda$ is the regularization coefficient and $(i<j)$ removes duplicated calculations. The optimization can be done with Stochastic Gradient Descent in order to be faster on sparse data, or with Alternating Least Squares to enable parallelization on dense data.

\subsubsection{Distribution estimation}

The original PrefNMF (Desarkar et al. 2012) computes the attractiveness of an item to a user by the product of their latent feature vectors which results in a scalar value, where the likelihoods of other possible values remain unknown. However, in order to be combined with $M R F$ models, we wish to have the distributions over a set of possible values. Therefore the Random Utility Models (McFadden 1980) and Ordinal Logistic Regression (McCullagh 1980) are applied to perform the conversion.

Random Utility Models (McFadden 1980) assume the existence of a latent utility $x_{u i}=$ $\mu_{u i}+\epsilon_{u i}$ that captures how much the user $u$ is interested in the item $i$, where $\mu_{u i}$ captures the interest and $\epsilon_{u i}$ is the random noise that follows the logistic distribution as in Koren and Sill (2011). The latent utility $x_{u i}$ is then generated from a logistic distribution centered at $\mu_{u i}$ with the scale parameter $s_{u i}$ proportional to the standard deviation:

$$
x_{u i} \sim \operatorname{Logi}\left(\mu_{u i}, s_{u i}\right)
$$

Recall that PrefNMF computes the attractiveness of an item to a user by the product of their latent feature vectors, thereby the internal score $\mu_{u i}$ can be substituted with the term $\mathbf{u}_{u}^{\top} \mathbf{v}_{i}$ : 


$$
x_{u i}=\mathbf{u}_{u}^{\top} \mathbf{v}_{i}+\epsilon_{u i}
$$

where $\mathbf{u}_{u}$ and $\mathbf{v}_{i}$ are, respectively, the latent feature vectors of the user $u$ and the item $i$.

Ordinal Logistic Regression (McCullagh 1980) is then used to convert the user-wise preferences $p_{u i}$ into ordinal values, which assumes that the preference $p_{u i}$ is chosen based on the interval to which the latent utility belongs:

$$
p_{u i}=l \text { if } x_{u i} \in\left(\theta_{l-1}, \theta_{l}\right] \text { for } l<L \text { and } p_{u i}=L \text { if } x_{u i}>\theta_{L-1}
$$

where $L$ is the number of ordinal levels and $\theta_{l}$ are the threshold values of interest. The probability of receiving a preference $l$ is therefore

$$
Q\left(p_{u i}=l \mid u, i\right)=\int_{\theta_{l-1}}^{\theta_{l}} P\left(x_{u i} \mid \theta\right) \mathrm{d} \theta=F\left(\theta_{l}\right)-F\left(\theta_{l-1}\right)
$$

where $F\left(\theta_{l}\right)$ is the cumulative logistic distribution evaluated at $\theta_{l}$ with standard deviation $s_{u i}$ :

$$
F\left(x_{u i} \leq l \mid \theta_{l}\right)=\frac{1}{1+\exp \left(-\frac{\theta_{u i l}-\mu_{u i}}{s_{u i}}\right)}
$$

The thresholds $\theta_{l}$ can be parameterized to depend on user or item. This paper employs the user-specific thresholds parameterization described in Koren and Sill (2011). Therefore a set of thresholds $\left\{\theta_{u l}\right\}_{l=1}^{L}$ is defined for each user $u$ to replace the thresholds $\theta_{\text {uil }}$ in Eq. 3.7, and is learned from data.

Given the learned ordinal distribution $Q\left(p_{u i} \mid u, i\right)$, not only can the preferences be predicted but also the confidence for each prediction. The ordinal distribution $Q\left(p_{u i} \mid u, i\right)$ captures the $G S$ information in a probabilistic form, and will be incorporated into $M R F$ and $C R F$ in Sect. 3.4. Note that the user preference is quantized into ordinal values in this process.

\subsection{Markov Random Fields}

Markov Random Fields (MRF) (Tran et al. 2007; Defazio and Caetano 2012) model a set of random variables having the Markov property with respect to an undirected graph $\mathcal{G}$. The undirected graph $\mathcal{G}$ consists of a set of vertices $\mathcal{V}$ connected by a set of edges $\mathcal{E}$ without orientation, where two vertices are neighborhoods of each other when connected. Each vertex in $\mathcal{V}$ encodes a random variable, and the Markov property implies that a variable is conditionally independent of others given its neighborhoods.

In this work, we use $M R F$ to model user-wise preferences and their interactions with respect to a set of undirected graphs. Specifically for each user $u$, there is a graph $\mathcal{G}_{u}$ with a set of vertices $\mathcal{V}_{u}$ and a set of edges $\mathcal{E}_{u}$. Each vertex in $\mathcal{V}_{u}$ represents a preference $p_{u i}$ of user $u$ on the item $i$. Note that the term preference is used instead of rating because in the new model the preference is not interpolated as absolute ratings but user-wise ordering of items. Each edge in $\mathcal{E}_{u}$ captures a relation between two preferences by the same user.

Two preferences are connected by an edge if they are given by the same user or on the same item, corresponding to the item-item and user-user correlations, respectively. Modeling these correlations is actually capturing the $L S$ information in the preferences. However, it is not easy to model two types of correlation at the same time as it will result in a large graph. Instead, we model the item-item and user-user correlations separately, and merge their predictions. Figure 1 shows an example of four graphs for user $u$, user $v$, item $i$, and item $j$. Note that vertices of different graphs are not connected directly, however, the weights are estimated across graphs when the edges correspond to the same correlation. For example, 

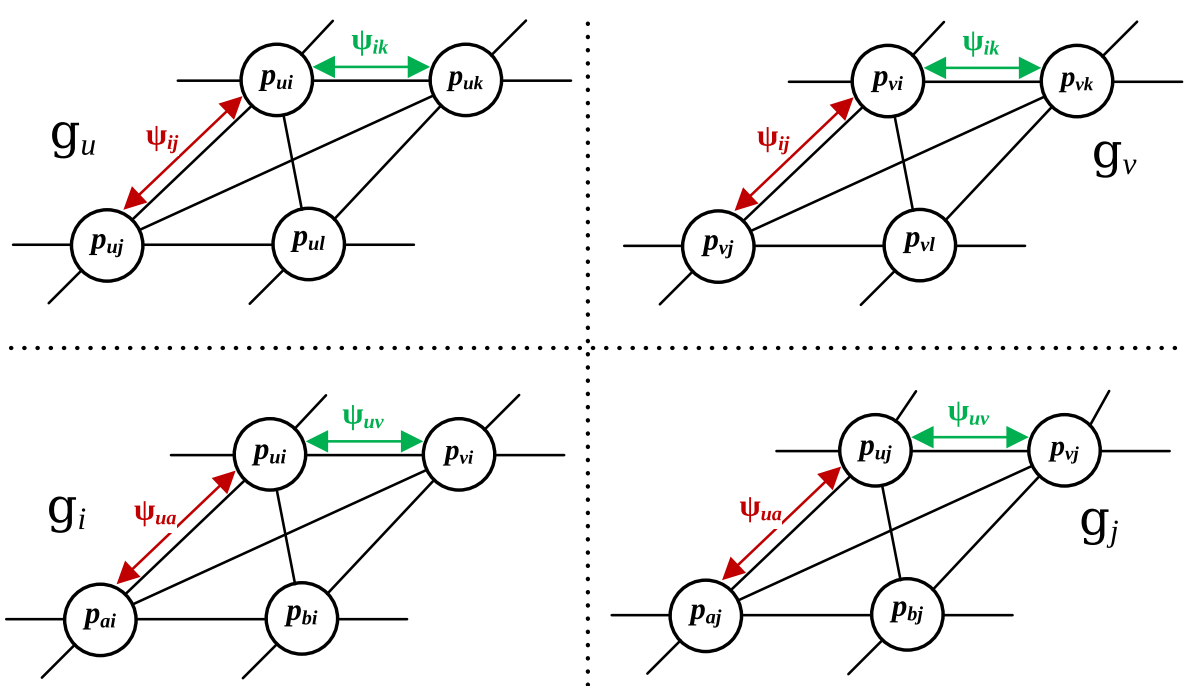

Fig. 1 Example of MRF graphs. $u, v, a$, and $b$ are users. $i, j, l$, and $k$ are items

the edge between $p_{u i}$ and $p_{u j}$ and the edge between $p_{v i}$ and $p_{v j}$ are associated with the same item-item correlation $\psi_{i j}$ between items $i$ and $j$.

Formally, let $\mathcal{I}_{u}$ be the set of all items evaluated by the user $u$ and $\mathcal{U}_{i}$ be the set of all users who rated the item $i$. Then denote $\mathbf{p}_{u}=\left\{p_{u i} \mid i \in \mathcal{I}_{u}\right\}$ to be the joint set of all preferences (the variables) expressed by user $u$, and $\mathbf{p}_{i}=\left\{p_{u i} \mid u \in \mathcal{U}_{i}\right\}$ to be the joint set of all preferences (the variables) rated on item $i$. Under this setting, the $M R F$ defines two distributions $P\left(\mathbf{p}_{u}\right)$ and $P\left(\mathbf{p}_{i}\right)$ over the graphs $\mathcal{G}_{u}$ and $\mathcal{G}_{i}$, respectively:

$$
\begin{aligned}
P\left(\mathbf{p}_{u}\right) & =\frac{1}{Z_{u}} \Psi_{u}\left(\mathbf{p}_{u}\right), P\left(\mathbf{p}_{i}\right)=\frac{1}{Z_{i}} \Psi_{i}\left(\mathbf{p}_{i}\right) \\
\Psi_{u}\left(\mathbf{p}_{u}\right) & =\prod_{(u i, u j) \in \mathcal{E}_{u}} \psi_{i j}\left(p_{u i}, p_{u j}\right), \Psi_{i}\left(\mathbf{p}_{i}\right)=\prod_{(u i, v i) \in \mathcal{E}_{i}} \psi_{u v}\left(p_{u i}, p_{v i}\right)
\end{aligned}
$$

where $Z_{u}$ and $Z_{i}$ are the normalization terms that ensure $\sum_{\mathbf{p}_{u}} P\left(\mathbf{p}_{u}\right)=1$ and $\sum_{\mathbf{p}_{i}} P\left(\mathbf{p}_{i}\right)=1$. The term $\psi(\cdot)$ is a positive function known as potential.

The potentials $\psi_{i j}\left(p_{u i}, p_{u j}\right)$ and $\psi_{u v}\left(p_{u i}, p_{v i}\right)$ capture the correlation between items $i$ and $j$ and correlation between users $u$ and $v$, respectively:

$$
\begin{aligned}
\psi_{i j}\left(p_{u i}, p_{u j}\right) & =\exp \left\{w_{i j} f_{i j}\left(p_{u i}, p_{u j}\right)\right\} \\
\psi_{u v}\left(p_{u i}, p_{v i}\right) & =\exp \left\{w_{u v} f_{u v}\left(p_{u i}, p_{v i}\right)\right\}
\end{aligned}
$$

where $f_{i j}(\cdot)$ and $f_{u v}(\cdot)$ are the feature functions to be designed shortly in Sect. 3.4.1, and $w_{i j}$ and $w_{u v}$ are the corresponding weights.

These correlation features capture the $L S$ information, while the weights realize the importance of each correlation feature. With the weights estimated from data, the unknown preference $p_{u i}$ can be predicted using item-item correlations:

$$
\hat{p}_{u i}=\underset{p_{u i} \in[-1,1]}{\arg \max } P\left(p_{u i} \mid \mathbf{p}_{u}\right)
$$


or using user-user correlations:

$$
\hat{p}_{u i}=\underset{p_{u i} \in[-1,1]}{\arg \max } P\left(p_{u i} \mid \mathbf{p}_{i}\right)
$$

where the confidences of the predictions can be measured by $P\left(p_{u i} \mid \mathbf{p}_{u}\right)$ and $P\left(p_{u i} \mid \mathbf{p}_{i}\right)$.

\subsection{Conditional random fields}

Despite user preferences, various side information including content (Balabanović and Shoham 1997; Basilico and Hofmann 2004), temporal dynamics (Koren 2010), and social relations (Ma et al. 2011) are also important in making quality recommendations. While there exist methods to incorporate side information, there is yet no $P R$-based method that can achieve this.

One advantage of Markov Random Fields is its extensibility, thus side information can be easily incorporated by extending the MRF to Conditional Random Fields (CRF). In MRF, the item-item and user-user correlations are modeled by a set of graphs, where each graph has a set of vertices representing the preferences. To incorporate side information, the $M R F$ is extended to $C R F$ by conditioning each vertex on a set of global observations $\mathbf{0}$, i.e., the side information in our context. Specifically, each user $u$ is associated with a set of attributes $\left\{\mathbf{0}_{u}\right\}$ such as gender and occupation. Similarly, each item $i$ is associated with a set of attributes $\left\{\mathbf{o}_{i}\right\}$ such as genres of movie. This side information is encoded as the set of global observations $\mathbf{o}=\left\{\left\{\mathbf{o}_{u}\right\},\left\{\mathbf{o}_{i}\right\}\right\}$. The graphs for item-item and user-user correlations conditioned on global observations are illustrated in Fig. 2.

Using the same settings as $M R F$ in Sect. 3.2, the $C R F$ models the conditional distributions $P\left(\mathbf{p}_{u} \mid \mathbf{o}\right)$ and $P\left(\mathbf{p}_{i} \mid \mathbf{o}\right)$ over the graphs $\mathcal{G}_{u}$ and $\mathcal{G}_{i}$, respectively:

$$
\begin{aligned}
& P\left(\mathbf{p}_{u} \mid \mathbf{o}\right)=\frac{1}{Z_{u}(\mathbf{o})} \Psi_{u}\left(\mathbf{p}_{u}, \mathbf{o}\right), P\left(\mathbf{p}_{i} \mid \mathbf{o}\right)=\frac{1}{Z_{i}(\mathbf{o})} \Psi_{i}\left(\mathbf{p}_{i}, \mathbf{o}\right) \\
& \Psi_{u}\left(\mathbf{p}_{u}, \mathbf{o}\right)=\prod_{(u i) \in \mathcal{V}_{u}} \psi_{u i}\left(p_{u i}, \mathbf{o}\right) \prod_{(u i, u j) \in \mathcal{E}_{u}} \psi_{i j}\left(p_{u i}, p_{u j}\right) \\
& \Psi_{i}\left(\mathbf{p}_{i}, \mathbf{o}\right)=\prod_{(u i) \in \mathcal{V}_{i}} \psi_{u i}\left(p_{u i}, \mathbf{o}\right) \prod_{(u i, v i) \in \mathcal{E}_{i}} \psi_{u v}\left(p_{u i}, p_{v i}\right)
\end{aligned}
$$

where $Z_{u}(\mathbf{o})$ and $Z_{i}(\mathbf{o})$ are the normalization terms that ensure $\sum_{\mathbf{p}_{u}} P\left(\mathbf{p}_{u} \mid \mathbf{o}\right)=1$ and $\sum_{\mathbf{p}_{i}} P\left(\mathbf{p}_{i} \mid \mathbf{o}\right)=1$.

The potentials $\psi_{i j}(\cdot)$ and $\psi_{u v}(\cdot)$ capture the item-item and user-user correlations in the same way as in Markov Random Fields, i.e., Eqs. 3.10 and 3.11. The potential $\psi_{u i}(\cdot)$ captures the global observations associated with the user $u$ and the item $i$ :

$$
\left.\psi_{u i}\left(p_{u i}, \mathbf{o}\right)=\exp \left\{\mathbf{w}_{u}^{\top} \mathbf{f}_{u}\left(p_{u i}, \mathbf{o}_{i}\right)+\mathbf{w}_{i}^{\top} \mathbf{f}_{i}\left(p_{u i}, \mathbf{o}_{u}\right)\right)\right\}
$$

where $\mathbf{f}_{u}$ and $\mathbf{f}_{i}$ are the features to be designed shortly in Sect. 3.4.1, and $\mathbf{w}_{u}$ and $\mathbf{w}_{i}$ are the corresponding weights realize the importance of each feature. With the weights estimated from data, the unknown preference $p_{u i}$ can be predicted using item-item correlations:

$$
\hat{p}_{u i}=\underset{p_{u i} \in[-1,1]}{\arg \max } P\left(p_{u i} \mid \mathbf{p}_{u}, \mathbf{o}\right)
$$

or using user-user correlations:

$$
\hat{p}_{u i}=\underset{p_{u i} \in[-1,1]}{\arg \max } P\left(p_{u i} \mid \mathbf{p}_{i}, \mathbf{o}\right)
$$




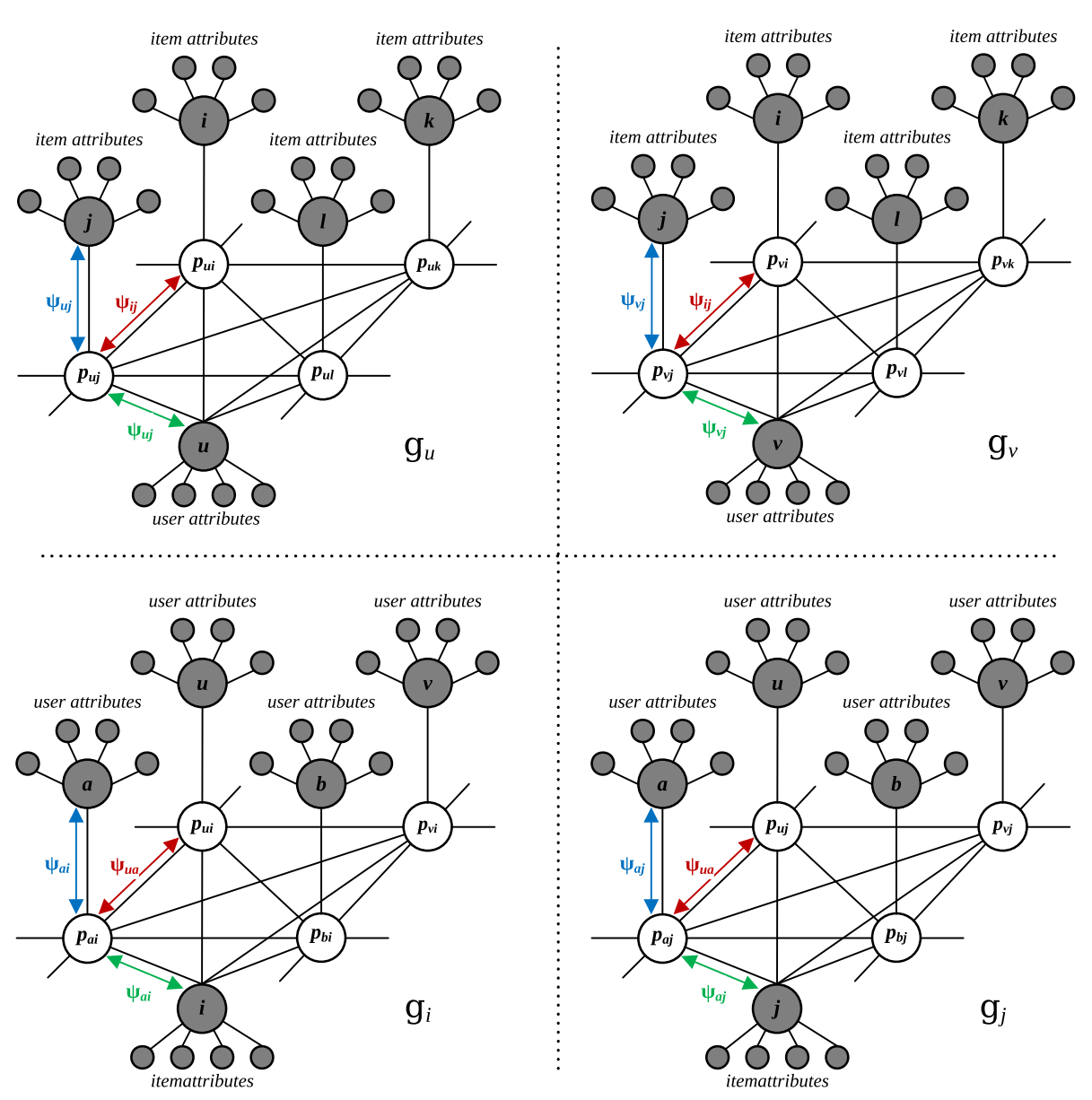

Fig. 2 Example of CRF graphs. $u, v, a$, and $b$ are users. $i, j, l$, and $k$ are items

where $P\left(p_{u i} \mid \mathbf{p}_{u}, \mathbf{o}\right)$ and $P\left(p_{u i} \mid \mathbf{p}_{i}, \mathbf{o}\right)$ give the confidence of the predictions.

\subsection{PrefCRF: unifying PrefNMF and CRF}

The $C R F$ model captures the $L S$ information by modeling item-item and user-user correlations under the framework of probabilistic graphical models. However, it employs the log-linear modeling as shown in Eqs. 3.10, 3.10, and 3.17, and therefore does not enable a simple treatment of $P R$. The PrefNMF model, on the other hand, can nicely model the $P R$ but is weak in capturing the $L S$ and side information. The complementarity between these two techniques enables the unified PrefCRF model to utilise the advantages of both models.

Essentially, the proposed PrefCRF model promotes the agreement between the GS discovered by the PrefNMF, the $L S$ discovered by the $M R F$, and the side information discovered by the $C R F$. More specifically, the PrefCRF model combines the item-item and user-user correlations (Eqs. 3.15, 3.16) and the ordinal distributions $Q\left(p_{u i} \mid u, i\right)$ over user-wise preferences obtained from Eq. 3.6: 


$$
\begin{gathered}
P\left(\mathbf{p}_{u} \mid \mathbf{o}\right) \propto \Psi_{u}\left(\mathbf{p}_{u}, \mathbf{o}\right) \prod_{p_{u i} \in \mathbf{p}_{u}} Q\left(p_{u i} \mid u, i\right) \\
P\left(\mathbf{p}_{i} \mid \mathbf{o}\right) \propto \Psi_{i}\left(\mathbf{p}_{i}, \mathbf{o}\right) \prod_{p_{u i} \in \mathbf{p}_{i}} Q\left(p_{u i} \mid u, i\right)
\end{gathered}
$$

where $\Psi_{u}$ is the potential function capturing the interaction among items evaluated by user $u$, and $\Psi_{i}$ is the potential function capturing the interaction among users who rated item $i$. Put together, the joint distribution $P\left(\mathbf{p}_{u}\right)$ for each user $u$ can be modeled as:

$$
P\left(\mathbf{p}_{u}\right) \propto \exp \left(\sum_{p_{u i}, p_{u j} \in \mathbf{p}_{u}} w_{i j} f_{i j}\left(p_{u i}, p_{u j}\right)+\sum_{(u i) \in \mathcal{V}_{u}} \psi_{u i}\left(p_{u i}, \mathbf{o}\right)\right) \prod_{p_{u i} \in \mathbf{p}_{u}} Q\left(p_{u i} \mid u, i\right)
$$

and the joint distribution $P\left(\mathbf{p}_{i}\right)$ for each item $i$ can be modeled as:

$$
P\left(\mathbf{p}_{i}\right) \propto \exp \left(\sum_{p_{u i}, p_{v i} \in \mathbf{p}_{i}} w_{u v} f_{u v}\left(p_{u i}, p_{v i}\right)+\sum_{(u i) \in \mathcal{V}_{i}} \psi_{u i}\left(p_{u i}, \mathbf{o}\right)\right) \prod_{p_{u i} \in \mathbf{p}_{i}} Q\left(p_{u i} \mid u, i\right)
$$

where there is a graph for each user or item but the weights are optimized by all users or all items.

\subsubsection{Feature design}

A feature is essentially a function $f$ of $n>1$ arguments that maps the $n$-dimensional input into the unit interval $f: \mathbb{R}^{n} \rightarrow[0,1]$. We design the following kinds of features:

Correlation features The item-item correlation is captured by the feature:

$$
f_{i j}\left(p_{u i}, p_{u j}\right)=g\left(\left|\left(p_{u i}-\bar{p}_{i}\right)-\left(p_{u j}-\bar{p}_{j}\right)\right|\right)
$$

and the user-user correlation is captured by the feature:

$$
f_{u v}\left(p_{u i}, p_{v i}\right)=g\left(\left|\left(p_{u i}-\bar{p}_{u}\right)-\left(p_{v i}-\bar{p}_{v}\right)\right|\right)
$$

where $g(\alpha)=1-\alpha /(L-1)$ normalizes feature values and $\alpha$ plays the role of deviation, where $L$ is the number of ordinal levels described in Sect. 3.1.2. The terms $\bar{p}_{i}, \bar{p}_{j}, \bar{p}_{i}$, and $\bar{p}_{j}$ are the item or user averages. The item-item correlation feature captures the intuition that correlated items should be ranked similarly by the same user after offsetting the goodness of each item. Similarly, the user-user correlation feature captures the intuition that correlated users should rate the same item similarly.

Attribute features Each user $u$ and item $i$ has a set of attributes $\mathbf{o}_{u}$ and $\mathbf{o}_{i}$, respectively. These attributes are mapped to preferences by the following features:

$$
\begin{aligned}
\mathbf{f}_{i}\left(p_{u i}\right) & =\mathbf{o}_{u} g\left(\left|\left(p_{u i}-\bar{p}_{i}\right)\right|\right) \\
\mathbf{f}_{u}\left(p_{u i}\right) & =\mathbf{o}_{i} g\left(\left|\left(p_{u i}-\bar{p}_{u}\right)\right|\right)
\end{aligned}
$$

where $\mathbf{f}_{i}$ models which users like the item $i$ and $\mathbf{f}_{u}$ models which classes of items the user $u$ likes.

Since one correlation feature exists for each possible pair of co-rated items, the number of correlation features can be large which makes the estimation slow to converge and less robust. Therefore we only keep the correlation features if strong item-item correlation or 
user-user correlation exists. Specifically, the strong correlation features $\mathbf{f}_{\text {strong }}$ are extracted based on the Pearson Correlation and a user-specified minimum correlation threshold. Note that the correlation is calculated based on the user-wise preferences generated from $P R$ thus the rule of using $P R$ as input is not violated.

\subsubsection{Parameter estimation}

In general, $M R F$-based models cannot be determined by standard maximum likelihood approaches, instead, approximation techniques are often used in practice such as Pseudolikelihood (Besag 1974) and Contrastive Divergence (CD) (Hinton 2002). The Pseudolikelihood leads to exact computation of the loss function and its gradient with respect to parameters, and thus is faster. The CD-based methods may, on the other hand, lead to better estimation given enough time. As the experiments involve different settings and large numbers of features, this study employs the Pseudo-likelihood technique to perform efficient parameter estimation by maximizing the regularized sum of log local likelihoods:

$$
\log \mathcal{L}(\mathbf{w})=\sum_{p_{u i} \in \Pi} \log P\left(p_{u i} \mid \mathbf{p}_{u}, \mathbf{o}\right)-\frac{1}{2 \sigma^{2}} \mathbf{w}^{\top} \mathbf{w}
$$

where $\mathbf{w}$ are the weights and $1 / 2 \sigma^{2}$ controls the regularization. To make the notation uncluttered, we write $\mathbf{p}_{u}$ instead of explicitly as $\mathbf{p}_{u} \backslash p_{u i}$. In this section we describe the parameter estimation of item-item correlations, where the user-user correlations can be estimated in the same way by replacing items with users.

The local likelihood in Eq. 3.27 is defined as:

$$
P\left(p_{u i} \mid \mathbf{p}_{u}, \mathbf{o}\right)=\frac{1}{Z_{u i}(\mathbf{o})} Q\left(p_{u i} \mid u, i\right) \psi_{u i}\left(p_{u i}, \mathbf{o}\right) \prod_{p_{u j} \in \mathbf{p}_{u}} \psi_{i j}\left(p_{u i}, p_{u j}\right)
$$

where $Z_{u i}(\mathbf{o})$ is the normalization term:

$$
Z_{u i}=\sum_{p_{u i}=l_{\min }}^{l_{\max }} Q\left(p_{u i} \mid u, i\right) \psi_{u i}\left(p_{u i}, \mathbf{o}\right) \prod_{p_{u j} \in \mathbf{p}_{u}} \psi_{i j}\left(p_{u i}, p_{u j}\right)
$$

where $l_{\min }$ is the first and $l_{\max }$ is the last interval, i.e., 1 and 3 in our settings.

To optimize the parameters, we use the stochastic gradient ascent procedure that updates the parameters by passing through the set of ratings of each user:

$$
\mathbf{w} \leftarrow \mathbf{w}+\eta \nabla \log \mathcal{L}(\mathbf{w})
$$

where $\eta$ is the learning rate. More specifically, for each $p_{u i}$ we update the attribute weights $\mathbf{w}_{o}=\left\{\mathbf{w}_{u}, \mathbf{w}_{i}\right\}$ and correlation weight $w_{i j}$ for each neighbor $p_{u j} \in \mathbf{p}_{u}$ using the gradient of the log pseudo-likelihood

$$
\begin{aligned}
\frac{\partial \log \mathcal{L}}{\partial \mathbf{w}_{o}} & =\mathbf{f}_{o}\left(p_{u i}, \mathbf{o}\right)-\sum_{p_{u i}=l_{\min }}^{l_{\max }} P\left(p_{u i} \mid \mathbf{p}_{u}, \mathbf{o}\right) \mathbf{f}_{o}\left(p_{u i}, \mathbf{o}\right)-\frac{w_{i}}{\sigma^{2}} \\
\frac{\partial \log \mathcal{L}}{\partial w_{i j}} & =f_{i j}\left(p_{u i}, p_{u j}\right)-\sum_{p_{u i}=l_{\min }}^{l_{\max }} P\left(p_{u i} \mid \mathbf{p}_{u}, \mathbf{o}\right) f_{i j}\left(p_{u i}, p_{u j}\right)-\frac{w_{i j}}{\sigma^{2}}
\end{aligned}
$$




\subsubsection{Item recommendation}

The ultimate goal of RecSys is often to rank the items and recommend the Top-N items to the user. To obtain the item rankings, the PrefCRF produces distributions over the user-wise preferences, which can be converted into point estimate:

Most Likely preference The preference can be determined by selecting the preference with the greatest mass in local likelihood:

$$
\hat{p}_{u i}=\underset{p_{u i}}{\arg \max } P\left(p_{u i} \mid \mathbf{p}_{u}, \mathbf{o}\right)
$$

where the local likelihood is given by Eq. 3.28. The local likelihood serves as a confidence measure.

Smoothed expectation When the prediction is not restricted to discrete values, the expectation can be used instead:

$$
\hat{p}_{u i}=\sum_{p_{u i}=l_{\min }}^{l_{\max }} p_{u i} P\left(p_{u i} \mid \mathbf{p}_{u}, \mathbf{o}\right)
$$

where $l$ refers to the intervals of user-wise preferences: from least to most preferred. Note that $l$ is limited to the simplest case of three intervals in our settings, but more intervals are possible.

The predictions by item-item correlation and user-user correlations can be merged by taking the mean value, and then items can be sorted and ranked accordingly. Finally, Algorithm 1 summarizes the learning and prediction procedures for the PrefCRF.

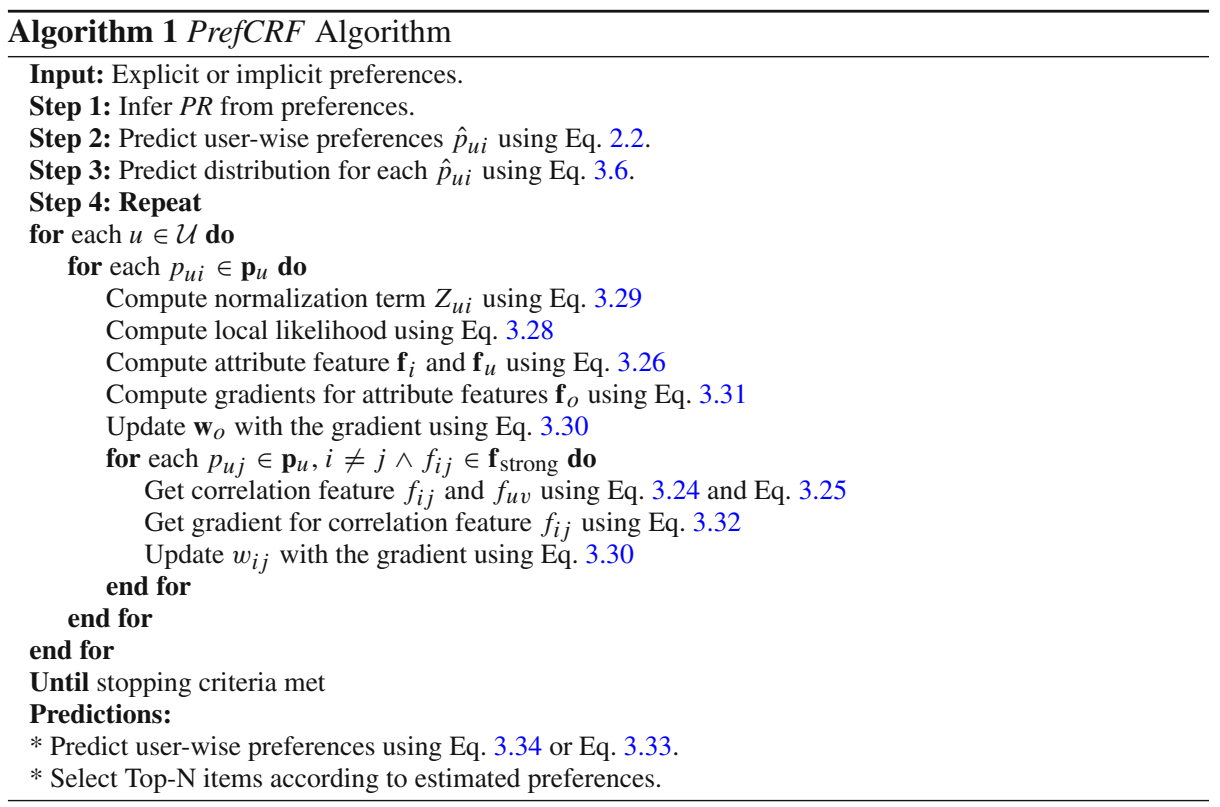




\subsubsection{Computational complexity}

We perform the computational complexity analysis on the PrefMRF and its underlying PrefNMF algorithms. Given $n$ users and $m$ items each with $d_{u}$ and $d_{i}$ preferences, respectively. Let us temporarily ignore the user-specified latent factors. Then the complexity of both PrefNMF and PrefMRF is $O\left(n d_{u}^{2}\right)$. However, in practice few items co-rated by the same user are strong neighbors of each other due to the correlation threshold defined in Sect. 3.4.1. As a result, the computation time of PrefMRF tends to be $O\left(n d_{u} c\right)$ where $c$ is a factor of the correlation threshold. It should be noted that Eq. 3.32 in the most inner loop of Algorithm 1 is not scaled with $d_{u}$ as the normalization term $Z_{u i}$ of the local likelihood is computed in the outer loop and reused for inner loop.

\section{Experiment and analysis}

To study the performance of the proposed PrefMRF and PrefCRF models, comparisons were done with the following representative algorithms: (a) K-Nearest Neighbors (KNN) (Resnick et al. 1994), which represents the methods exploiting the $L S$ from absolute ratings; (b) Non-negative Matrix Factorization (NMF) (Koren et al. 2009), which represents the methods exploiting the GS from absolute ratings; (c) Preference Relation-based KNN (PrefKNN) (Brun et al. 2010), which exploits the LS from PR; (d) Preference Relation-based $N M F$ (PrefNMF) (Desarkar et al. 2012), which exploits the $G S$ from $P R$.

\subsection{Experimental settings}

\subsubsection{Datasets}

Ideally, the experiments should be conducted on datasets that contain user preferences in two forms: $P R$ and absolute ratings. Unfortunately no such dataset is publicly available at the moment, therefore we choose to compile the rating-based datasets into the form of $P R$. We use the same conversion method as in Desarkar et al. (2012) by comparing the ratings of each ordered pair of items co-rated by the same user. For example, 1 is assigned to the $P R$ $\pi_{u i j}$ if $p_{u i}>p_{u j} ; 0$ is assigned if $p_{u i}<p_{u j}$, and 0.5 is assigned if $p_{u i}=p_{u j}$. Though this experiment converts ratings into PR, it should be noted that the proposed PR-based models are designed to handle implicit preferences such as page views, mouse clicks, and time stayed, which can be converted into PR. In such cases, the pointwise models are not applicable.

Experiments were conducted on two datasets: the MovieLens- $1 \mathrm{M}^{1}$ and the EachMovie ${ }^{2}$ datasets. The MovieLens-1M dataset contains more than 1 million ratings by 6040 users on 3900 movies. The EachMovie dataset contains 2.8 million ratings by 72,916 users on 1628 movies. The minimum rating is 1 and we cap the maximum at 5 for both datasets. The impact of side information is studied on the MovieLens-1M dataset which provides gender, age, and occupation information about users and genres of movies.

For a reliable and fair comparison, each dataset is split into train and test sets, and the following settings are aligned to related work (Weimer et al. 2007). As the sparsity levels differ between the MovieLens-1M and the EachMovie datasets, different numbers of ratings are reserved for training and the rest for testing. Specifically, for each user in the MovieLens-

\footnotetext{
1 http://grouplens.org/datasets/movielens.

2 http://grouplens.org/datasets/eachmovie.
} 
$1 \mathrm{M}$ we randomly select $N=30,40,50,60$ ratings for training, and put the rest for testing. Some users do not have enough ratings thus were excluded from experiments. The EachMovie has less items but much more users than MovieLens-1M, therefore it is safe to remove some less active users and we set $N=70,80,90,100$ to investigate the performance on a dense dataset.

\subsubsection{Evaluation metrics}

Traditional recommender systems aim to optimize $R M S E$ or $M A E$ which emphasizes on absolute ratings. However, the ultimate goal of recommender systems is usually to obtain the ranking of items (Koren and Sill 2011), where good performance on RMSE or MAE may not be translated into good ranking results (Koren and Sill 2011). Therefore, we employ two evaluation metrics: Normalized Cumulative Discounted Gain@T (NDCG@T) (Järvelin and Kekäläinen 2002) which is popular in academia, and Mean Average Precision@T (MAP@T) (Chapelle et al. 2009) which is popular in contests. ${ }^{3}$ Among them, the NDCG@T metric is defined as

$$
\mathrm{NDCG} @ T=\frac{1}{K(T)} \sum_{t=1}^{T} \frac{2^{r_{t}}-1}{\log _{2}(t+1)}
$$

where $r_{t}$ is the relevance judgment of the item at position $t$, and $K(T)$ is a normalization constant.The MAP@T metric is defined as

$$
\mathrm{MAP} @ T=\frac{1}{\left|\mathcal{U}_{\text {test }}\right|} \sum_{u \in \mathcal{U}_{\text {test }}} \sum_{t=1}^{T} \frac{P_{u}(t)}{\min \left(m_{u}, t\right)}
$$

where $m_{u}$ is the number of relevant items to user $u$, and $P_{u}(t)$ is user $u$ 's precision at position $t$. Both metrics are normalized to $[0,1]$, and a higher value indicates better performance.

These metrics, together with other ranking-based metrics, require a set of relevant items to be defined in the test set such that the predicted rankings can be evaluated. The relevant items can be defined in different ways. In this paper, we follow the same selection criteria used in the related work (Koren 2008; Brun et al. 2010) to consider items with the highest ratings as relevant.

\subsubsection{Parameter setting}

For a fair comparison, we fix the number of latent factors to 50 for all algorithms, the same as in related work (Cremonesi et al. 2010). The number of neighbors for $K N N$ algorithms is set to 50. We vary the minimum correlation threshold to examine the performance with different numbers of features. Different values of the regularization coefficient are also tested.

\subsection{Results and analysis}

We first compare the performance of the proposed PrefMRF and PrefCRF models with four related models: $K N N, N M F$, PrefKNN, and PrefNMF, where the PrefNMF is the targeted model, and then investigate the impact of parameter settings.

3 KDD Cup 2012 and Facebook Recruiting Competition. 
Table 3 Mean results and standard deviation over ten runs on MovieLens-1M dataset

\begin{tabular}{|c|c|c|c|c|}
\hline \multicolumn{5}{|c|}{ Given 30} \\
\hline Algorithm & NDCG@5 & NDCG@10 & MAP@5 & MAP@10 \\
\hline UserKNN & $0.3969 \pm 0.0020$ & $0.4081 \pm 0.0029$ & $0.2793 \pm 0.0021$ & $0.2744 \pm 0.0025$ \\
\hline NMF & $0.5232 \pm 0.0057$ & $0.5195 \pm 0.0040$ & $0.3866 \pm 0.0055$ & $0.3549 \pm 0.0037$ \\
\hline PrefKNN & $0.3910 \pm 0.0044$ & $0.4048 \pm 0.0038$ & $0.2745 \pm 0.0043$ & $0.2720 \pm 0.0037$ \\
\hline PrefNMF & $0.5729 \pm 0.0049$ & $0.5680 \pm 0.0041$ & $0.4387 \pm 0.0046$ & $0.3992 \pm 0.0033$ \\
\hline PrefMRF & $0.6020 \pm 0.0050$ & $0.5934 \pm 0.0039$ & $0.4721 \pm 0.0050$ & $0.4244 \pm 0.0036$ \\
\hline PrefCRF & $0.6316 \pm 0.0076$ & $0.5966 \pm 0.0028$ & $0.6254 \pm 0.0073$ & $0.4245 \pm 0.0028$ \\
\hline \multicolumn{5}{|c|}{ Given 40} \\
\hline Algorithm & NDCG@5 & NDCG@10 & MAP@5 & MAP@10 \\
\hline UserKNN & $0.4108 \pm 0.0040$ & $0.4252 \pm 0.0036$ & $0.2936 \pm 0.0036$ & $0.2877 \pm 0.0034$ \\
\hline NMF & $0.5323 \pm 0.0050$ & $0.5291 \pm 0.0034$ & $0.3976 \pm 0.0045$ & $0.3631 \pm 0.0035$ \\
\hline PrefKNN & $0.4122 \pm 0.0024$ & $0.4283 \pm 0.0024$ & $0.2944 \pm 0.0023$ & $0.2904 \pm 0.0023$ \\
\hline PrefNMF & $0.5773 \pm 0.0037$ & $0.5732 \pm 0.0028$ & $0.4437 \pm 0.0041$ & $0.4019 \pm 0.0032$ \\
\hline PrefMRF & $0.6215 \pm 0.0029$ & $0.6140 \pm 0.0023$ & $0.4844 \pm 0.0025$ & $0.4420 \pm 0.0020$ \\
\hline PrefCRF & $0.6435 \pm 0.0064$ & $0.6092 \pm 0.0023$ & $0.6420 \pm 0.0062$ & $0.4392 \pm 0.0021$ \\
\hline \multicolumn{5}{|c|}{ Given 50} \\
\hline Algorithm & NDCG@5 & NDCG@10 & MAP@5 & MAP@10 \\
\hline UserKNN & $0.4273 \pm 0.0040$ & $0.4424 \pm 0.0027$ & $0.3078 \pm 0.0038$ & $0.3015 \pm 0.0026$ \\
\hline NMF & $0.5360 \pm 0.0041$ & $0.5326 \pm 0.0036$ & $0.4010 \pm 0.0040$ & $0.3669 \pm 0.0025$ \\
\hline PrefKNN & $0.4326 \pm 0.0027$ & $0.4483 \pm 0.0030$ & $0.3125 \pm 0.0024$ & $0.3070 \pm 0.0022$ \\
\hline PrefNMF & $0.5761 \pm 0.0067$ & $0.5745 \pm 0.0035$ & $0.4424 \pm 0.0064$ & $0.4019 \pm 0.0033$ \\
\hline PrefMRF & $0.6248 \pm 0.0053$ & $0.6172 \pm 0.0032$ & $0.4896 \pm 0.0053$ & $0.4460 \pm 0.0027$ \\
\hline PrefCRF & $0.6648 \pm 0.0055$ & $0.6158 \pm 0.0018$ & $0.6580 \pm 0.0059$ & $0.4471 \pm 0.0024$ \\
\hline \multicolumn{5}{|c|}{ Given 60} \\
\hline Algorithm & NDCG@5 & NDCG@10 & MAP@5 & MAP@10 \\
\hline UserKNN & $0.4480 \pm 0.0044$ & $0.4622 \pm 0.0035$ & $0.3266 \pm 0.0036$ & $0.3163 \pm 0.0027$ \\
\hline NMF & $0.5462 \pm 0.0068$ & $0.5409 \pm 0.0063$ & $0.4109 \pm 0.0069$ & $0.3734 \pm 0.0055$ \\
\hline PrefKNN & $0.4526 \pm 0.0062$ & $0.4689 \pm 0.0039$ & $0.3301 \pm 0.0051$ & $0.3223 \pm 0.0033$ \\
\hline PrefNMF & $0.5756 \pm 0.0062$ & $0.5733 \pm 0.0048$ & $0.4409 \pm 0.0059$ & $0.4007 \pm 0.0037$ \\
\hline PrefMRF & $0.6422 \pm 0.0037$ & $0.6301 \pm 0.0037$ & $0.5112 \pm 0.0035$ & $0.4600 \pm 0.0026$ \\
\hline PrefCRF & $0.6772 \pm 0.0074$ & $0.6242 \pm 0.0018$ & $0.6715 \pm 0.0072$ & $0.4536 \pm 0.0016$ \\
\hline
\end{tabular}

Best results are given in bold

\subsubsection{Comparison on Top- $N$ recommendation}

Comparison of these algorithms is conducted by measuring the $N D C G$ and the MAP metrics on Top-N recommendation tasks. Each experiment is repeated ten times with different random seeds and we report the mean results with standard deviations on the MovieLens-1M dataset in Table 3 and the EachMovie dataset in Table 4. Note that only the MovieLens-1M dataset has side information which is used by the PrefCRF model. The PrefMRF as well as other 
Table 4 Mean results and standard deviation over ten runs on EachMovie dataset withouut side information

\begin{tabular}{|c|c|c|c|c|}
\hline \multirow[b]{2}{*}{ Algorithm } & \multicolumn{3}{|c|}{ Given 70} & \multirow[b]{2}{*}{ MAP@10 } \\
\hline & NDCG@5 & NDCG@10 & MAP@5 & \\
\hline UserKNN & $0.7088 \pm 0.0020$ & $0.7115 \pm 0.0015$ & $0.6012 \pm 0.0027$ & $0.5767 \pm 0.0017$ \\
\hline NMF & $0.7581 \pm 0.0022$ & $0.7577 \pm 0.0017$ & $0.6524 \pm 0.0026$ & $0.6225 \pm 0.0020$ \\
\hline PrefKNN & $0.7260 \pm 0.0022$ & $0.7307 \pm 0.0018$ & $0.6197 \pm 0.0020$ & $0.5990 \pm 0.0016$ \\
\hline PrefNMF & $0.7408 \pm 0.0033$ & $0.7348 \pm 0.0039$ & $0.6330 \pm 0.0035$ & $0.5800 \pm 0.0038$ \\
\hline PrefMRF & $0.8317 \pm 0.0032$ & $0.8245 \pm 0.0029$ & $0.7512 \pm 0.0039$ & $0.6921 \pm 0.0034$ \\
\hline \multicolumn{5}{|c|}{ Given 80} \\
\hline Algorithm & NDCG@5 & NDCG@10 & MAP@5 & MAP@10 \\
\hline UserKNN & $0.7146 \pm 0.0018$ & $0.7168 \pm 0.0017$ & $0.6070 \pm 0.0021$ & $0.5825 \pm 0.0019$ \\
\hline $\mathrm{NMF}$ & $0.7636 \pm 0.0021$ & $0.7638 \pm 0.0018$ & $0.6583 \pm 0.0025$ & $0.6286 \pm 0.0018$ \\
\hline PrefKNN & $0.7337 \pm 0.0028$ & $0.7377 \pm 0.0018$ & $0.6271 \pm 0.0029$ & $0.6057 \pm 0.0021$ \\
\hline PrefNMF & $0.7422 \pm 0.0036$ & $0.7319 \pm 0.0040$ & $0.6329 \pm 0.0039$ & $0.5774 \pm 0.0033$ \\
\hline PrefMRF & $0.8364 \pm 0.0036$ & $0.8232 \pm 0.0030$ & $0.7553 \pm 0.0038$ & $0.6991 \pm 0.0032$ \\
\hline \multicolumn{5}{|c|}{ Given 90} \\
\hline Algorithm & NDCG@5 & NDCG@10 & MAP@5 & MAP@10 \\
\hline UserKNN & $0.7191 \pm 0.0022$ & $0.7279 \pm 0.0028$ & $0.6120 \pm 0.0021$ & $0.5933 \pm 0.0013$ \\
\hline $\mathrm{NMF}$ & $0.7712 \pm 0.0039$ & $0.7692 \pm 0.0033$ & $0.6663 \pm 0.0043$ & $0.6431 \pm 0.0034$ \\
\hline PrefKNN & $0.7418 \pm 0.0028$ & $0.7421 \pm 0.0015$ & $0.6357 \pm 0.0030$ & $0.6192 \pm 0.0020$ \\
\hline PrefNMF & $0.7456 \pm 0.0031$ & $0.7358 \pm 0.0038$ & $0.6357 \pm 0.0040$ & $0.5819 \pm 0.0036$ \\
\hline PrefMRF & $0.8394 \pm 0.0035$ & $0.8249 \pm 0.0032$ & $0.7474 \pm 0.0037$ & $0.7046 \pm 0.0032$ \\
\hline \multicolumn{5}{|c|}{ Given 100} \\
\hline Algorithm & NDCG@5 & NDCG@10 & MAP@5 & MAP@10 \\
\hline UserKNN & $0.7279 \pm 0.0028$ & $0.7277 \pm 0.0015$ & $0.6238 \pm 0.0032$ & $0.5973 \pm 0.0021$ \\
\hline NMF & $0.7741 \pm 0.0030$ & $0.7717 \pm 0.0028$ & $0.6719 \pm 0.0034$ & $0.6411 \pm 0.0030$ \\
\hline PrefKNN & $0.7505 \pm 0.0019$ & $0.7511 \pm 0.0012$ & $0.6478 \pm 0.0020$ & $0.6231 \pm 0.0014$ \\
\hline PrefNMF & $0.7391 \pm 0.0033$ & $0.7298 \pm 0.0034$ & $0.6318 \pm 0.0039$ & $0.5761 \pm 0.0039$ \\
\hline PrefMRF & $0.8418 \pm 0.0031$ & $\mathbf{0 . 8 2 7 7} \pm \mathbf{0 . 0 0 3 0}$ & $0.7546 \pm 0.0038$ & $0.7063 \pm 0.0036$ \\
\hline
\end{tabular}

Best results are given in bold

models are based on only preferences data. We also report the NDCG and MAP values by varying the position $T$ (i.e., how many items to recommend) in Fig. 3 for the MovieLens-1M dataset and in Fig. 4 for the EachMovie-1M dataset. The following observations can be made based on the results.

Firstly, the KNN and the PrefKNN methods didn't perform well on MovieLens-1M comparing with Matrix Factorization based methods. One possible reason is that predictions are made based only on the neighbors, and as a result too much information has been ignored especially when the dataset is large. However, the performance of $K N N$-based methods has improved on the EachMovie dataset as we reserved more ratings for training, i.e., better neighbors can be found for prediction. 


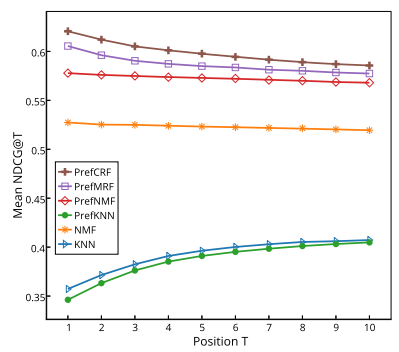

(a)

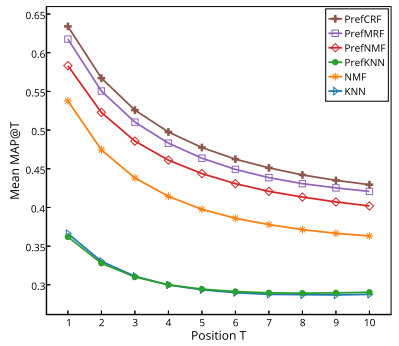

(d)

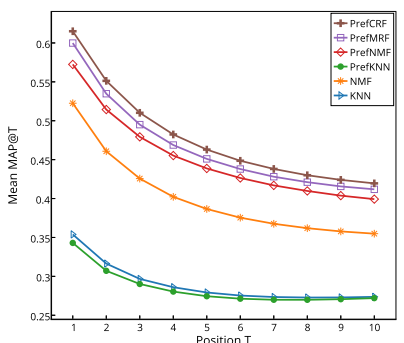

(b)

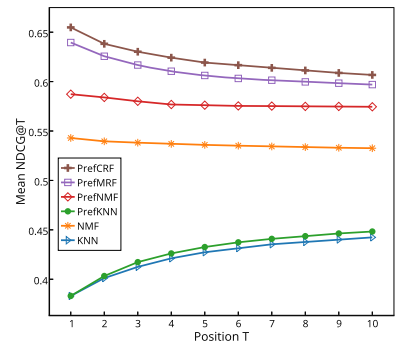

(e)

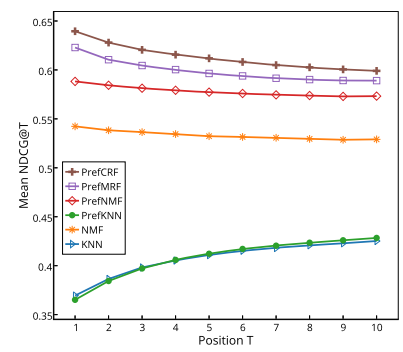

(c)

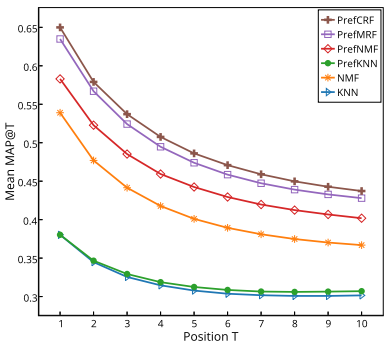

(f)

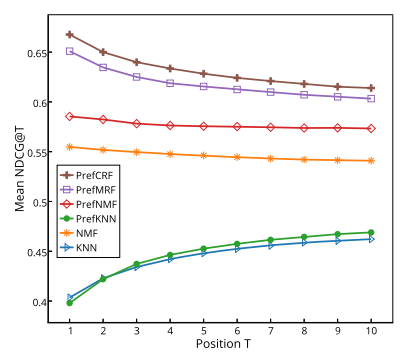

(g)

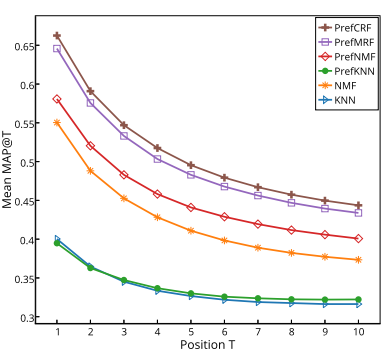

(h)

Fig. 3 Performance of different position $T$ on MovieLens-1M dataset. a NDCG@T (Given 30), b MAP@T (Given 30), c NDCG@T (Given 40), d MAP@T (Given 40), e NDCG@T (Given 50), f MAP@T (Given 50), g NDCG@T (Given 60), h MAP@T (Given 60)

Secondly, PrefNMF outperforms NMF on the MovieLens-1M dataset which is consistent with the results reported in Desarkar et al. (2012). However, PreNMF does not perform well on EachMovie where its performance is only slightly better than user-based $K N N$. The reason behind could be that EachMovie is much denser than the MovieLens-1M dataset, which makes the number of $P R$ huge and difficult to tune optimal parameters. Besides, we observe that PrefNMF in general only achieves a slight improvement with more training data and even drops a bit with Given 60. Similarly for the EachMovie dataset. With these observations, it appears that for a given number of users, the PrefNMF can be trained reasonably well with fewer data.

Finally, the proposed PrefMRF and PrefCRF have made further improvements over PrefNMF on both datasets through capturing both $L S$ and $G S$, as well as exploiting side information. From Fig. 3 we can see that the algorithms stabilized around position 10 and PrefMRF and PrefCRF consistently deliver better performance than others. It should be noted that the performance of PrefMRF and PrefCRF rely on their underlying model that captures 


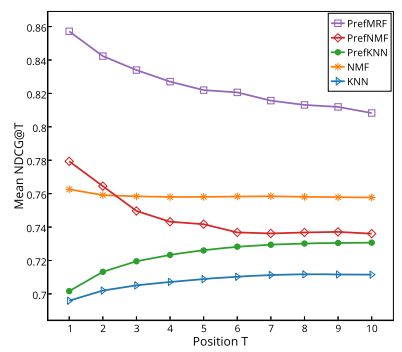

(a)

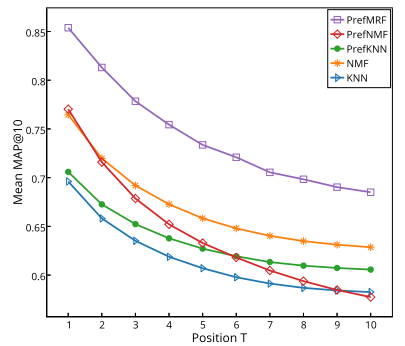

(d)

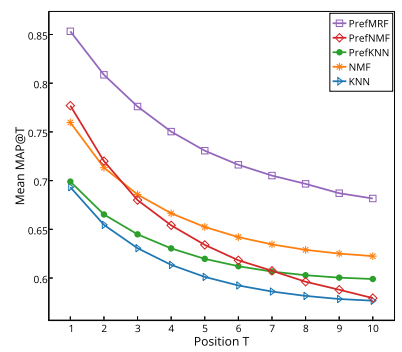

(b)

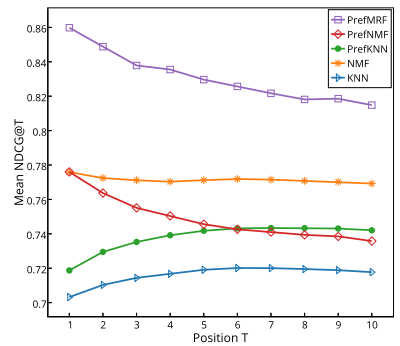

(e)

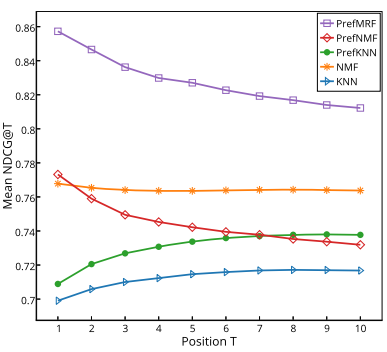

(c)

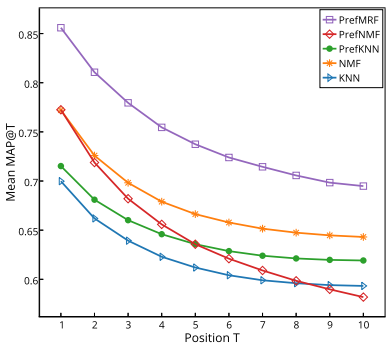

(f)

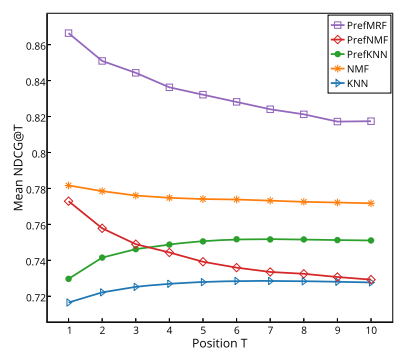

(g)

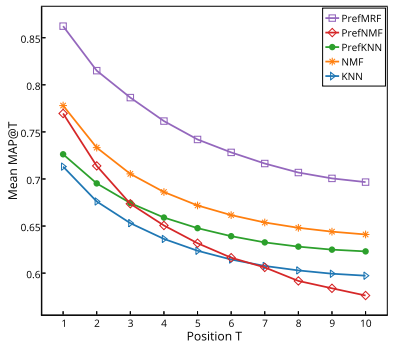

(h)

Fig. 4 Performance of different position $T$ on EachMovie dataset. a NDCG@T (Given 70), b MAP@T (Given 70), c NDCG@T (Given 80),d MAP@T (Given 80), e NDCG@T (Given 90), f MAP@T (Given 90), g NDCG@T (Given 100), h MAP@T (Given 100)

Table 5 Paired $t$-test for PrefMRF and PrefNMF

\begin{tabular}{lllllll}
\hline Settings & & & & \multicolumn{3}{l}{$t$-test statistics } \\
Dataset & Sparsity & Metric & & $d f$ & $\mathrm{t}$ & $p$ value \\
\hline MovieLens & Given 60 & NDCG@10 & 9 & 16.6218 & $<0.00001$ \\
MovieLens & Given 60 & MAP@10 & 9 & 23.5517 & $<0.00001$ \\
EachMovie & Given 100 & NDCG@10 & 9 & 72.4189 & $<0.00001$ \\
EachMovie & Given 100 & MAP@10 & 9 & 72.1346 & $<0.00001$ \\
\hline
\end{tabular}

the GS. In other words, the performance may vary when the PrefNMF is replaced with other alternative methods such as Liu et al. (2009).

To confirm the improvements, a paired $t$-test (two-tailed) with a significance level of 95\% has been applied to the best PrefMRF and the second best PrefNMF. Results shown 


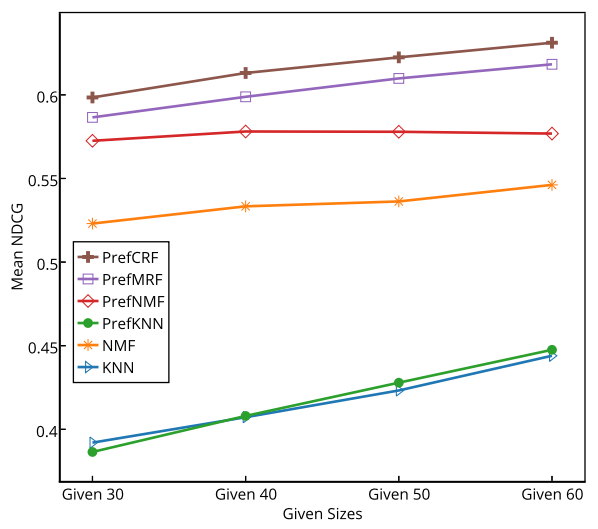

(a)

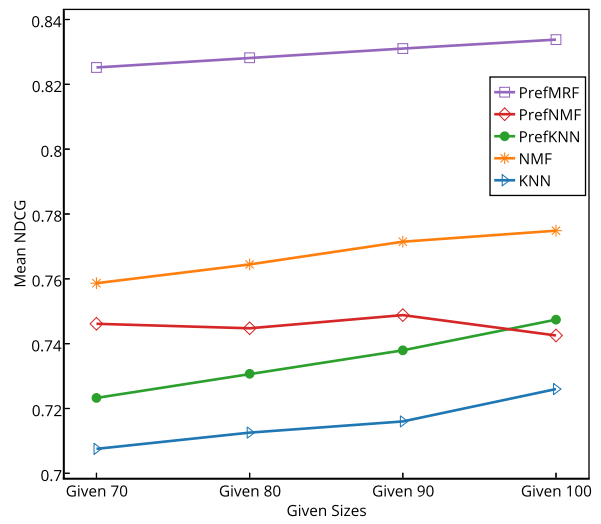

(c)

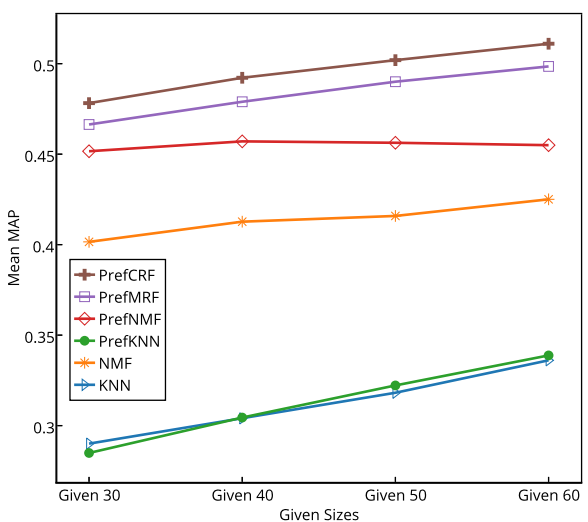

(b)

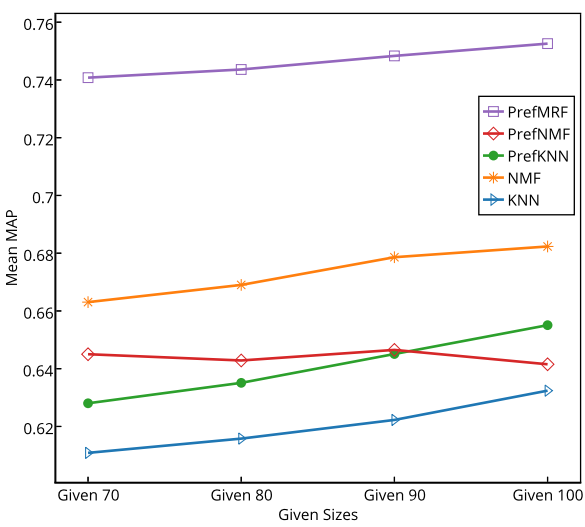

(d)

Fig. 5 Impact of sparsity levels. a Mean NDCG by training set sizes on MovieLens-1M dataset. b Mean MAP by training set sizes on MovieLens-1M dataset. c Mean NDCG by training set sizes on EachMovie dataset. d Mean MAP by training set sizes on EachMovie dataset

in Table 5 confirm that the performance of models with and without capturing the $L S$ is statistically significant.

\subsubsection{Performance on various data sparsity levels}

To thoroughly examine the performance of these algorithms, we compare their performance under different settings of density levels by varying the training set sizes: from Given 30 to Given 60 on MovieLens-1M dataset, and from Given 70 to Given 100 on EachMovie dataset. Results are plotted in Fig. 5. It can be observed that in general more training data results in better performance, and this is particularly true for $L S$-based models. However, PrefNMF does not gain much benefit from more data and even performs slightly worse in Given 60 . The PrefMRF on the other hand consistently gains performance from more data as the $L S$ information can be better captured. 


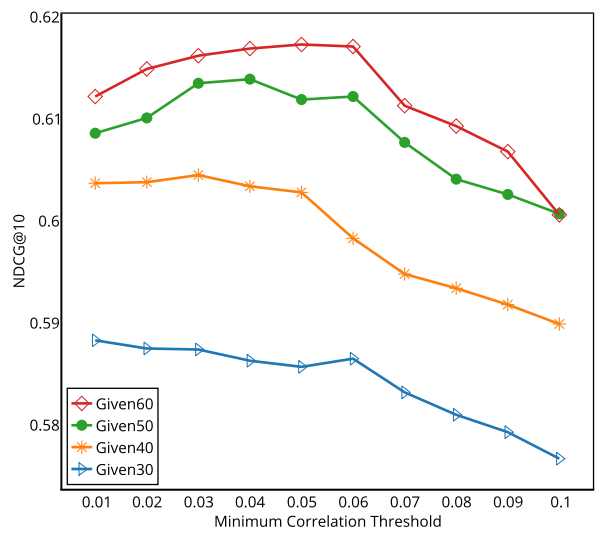

(a)

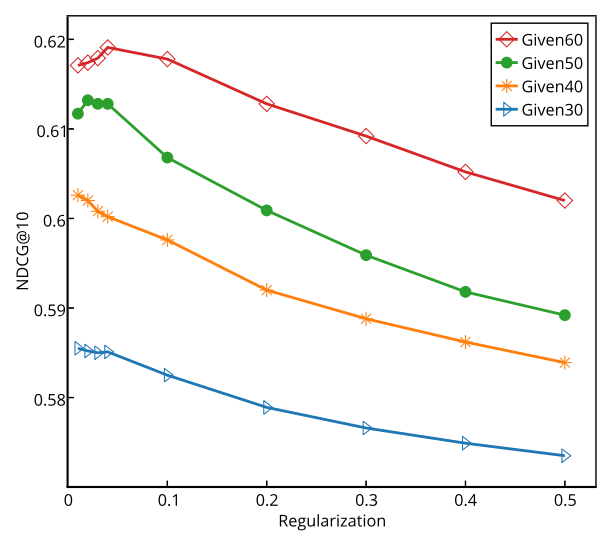

(b)

Fig. 6 Impact of Parameters (MovieLens-1M). a NDCG@10 by threshold. b NDCG@10 by regularization

\subsubsection{Impact of minimum correlation threshold}

As described in Sect. 3.4.1, a minimum correlation threshold is required to control the number of features in the PrefMRF model. By default, each pair of co-rated items has a feature which results in a large number of features. However, many of these features are useless if the itemitem correlations are weak. To make the model more robust and with faster convergence, a minimum correlation threshold is applied to remove weak features. Specifically, the feature is removed if two items has a correlation measured by Pearson correlation less than the threshold. Results are plotted in Fig. 6a.

It can be observed that a smaller correlation threshold delivers better performance, however, the number of features will also increase. To balance the performance and computation time, it is wise to select a moderate level of threshold depending on the dataset.

\subsubsection{Impact of regularization coefficient}

As the number of features in PrefMRF can be large, the model might be prone to over-fitting. Therefore, we investigate the impact of regularization settings as plotted in Fig. $6 \mathrm{~b}$.

We observe that the performance is better when a small regularization penalty applies. In other words, the PrefMRF can generalize reasonably well without too much regularization. This can be explained as the weights of item-item correlations are not user-specific but shared by all users, thus they cannot over-fit every user perfectly.

\section{Conclusions and future work}

In this paper we presented the PrefMRF model, which takes advantage of both the representational power of the $M R F$ and the ease of modeling preference relations by the PrefNMF. To the best of our knowledge, there was no $P R$-based method that can capture both $L S$ and $G S$, until the PrefMRF model was proposed in this work. In addition, side information can be easily incorporated by extending the PrefMRF model to the PrefCRF model. Experimental results on public datasets demonstrate that both types of interactions have been properly cap- 
tured by PrefMRF, and significantly improved Top-N recommendation performance has been achieved. In addition, the PrefMRF model provides a generic interface for unifying various models other than the PrefNMF used in this paper. In other words, a model that captures the $G S$ should be able to take advantage of PrefMRF to capture $L S$ as well by substituting their predictions into the formulas in Sect. 3.1.2.

For future work, we would like to work on two directions. First, the computation efficiency of $P R$-based matrix factorization needs to be improved given that the number of preference relations is usually much larger than absolute ratings. This is feasible as each user has his/her own set of preference relations, thus the learning process can be parallelized. Secondly, it would be interesting to see how $P R$-based methods perform on real implicit preferences dataset, such as page views and mouse clicks.

Acknowledgements The authors would like to thank the editors and the anonymous reviewers for their insightful comments and suggestions, which have greatly improved this manuscript. This work was partially supported by the National Science Foundation of China (61273301), the Collaborative Innovation Center of Novel Software Technology and Industrialization, and Guangxi Key Laboratory of Trusted Software (No. KX201528).

\section{References}

Balabanović, M., \& Shoham, Y. (1997). Fab: content-based, collaborative recommendation. Communications of the ACM, 40(3), 66-72.

Basilico, J., \& Hofmann, T. (2004). Unifying collaborative and content-based filtering. In Proceedings of ICML (pp. 65-72). ACM.

Besag, J. (1974). Spatial interaction and the statistical analysis of lattice systems. Journal of the Royal Statistical Society, Series B, 36(2), 192-236.

Brun, A., Hamad, A., Buffet, O., \& Boyer, A. (2010). Towards preference relations in recommender systems. In Preference learning (PL 2010) ECML/PKDD 2010 workshop.

Chapelle, O., Metlzer, D., Zhang, Y., \& Grinspan, P. (2009). Expected reciprocal rank for graded relevance. In Proceedings of CIKM (pp. 621-630). ACM.

Cohen, W. W., Schapire, R. E., \& Singer, Y. (1999). Learning to order things. Journal of Artificial Intelligence Research, 10(1), 243-270.

Cremonesi, P., Koren, Y., \& Turrin, R. (2010). Performance of recommender algorithms on top-n recommendation tasks. In Proceedings of the 4th ACM conference on recommender systems (pp. 39-46). ACM.

Defazio, A., \& Caetano, T. (2012). A graphical model formulation of collaborative filtering neighbourhood methods with fast maximum entropy training. In Proceedings of ICML (pp. 265-272).

Desarkar, M. S., Sarkar, S., \& Mitra. P. (2010). Aggregating preference graphs for collaborative rating prediction. In Proceedings of the 4th ACM conference on Recommender systems (pp. 21-28). ACM.

Desarkar, M. S., Saxena, R., \& Sarkar, S. (2012). Preference relation based matrix factorization for recommender systems. In International conference on user modeling, adaptation, and personalization (pp. 63-75). Springer.

Freund, Y., Iyer, R., Schapire, R. E., \& Singer, Y. (2003). An efficient boosting algorithm for combining preferences. Journal of Machine Learning Research, 4, 933-969.

Fürnkranz, J., \& Hüllermeier, E. (2003). Pairwise preference learning and ranking. In European conference on machine learning (pp. 145-156). Springer.

Fürnkranz, J., \& Hüllermeier, E. (2010). Preference learning. Berlin: Springer.

Gantner, Z., Drumond, L., Freudenthaler, C., Rendle, S., \& Schmidt-Thieme, L. (2010). Learning attributeto-feature mappings for cold-start recommendations. In Proceedings of ICDM (pp. 176-185). IEEE.

Hinton, G. (2002). Training products of experts by minimizing contrastive divergence. Neural Computation, 14(8), 1771-1800.

Järvelin, K., \& Kekäläinen, J. (2002). Cumulated gain-based evaluation of ir techniques. ACM Transactions on Information Systems, 20(4), 422-446.

Jin, R., Si, L., \& Zhai, C. (2002). Preference-based graphic models for collaborative filtering. In Proceedings of UAI (pp. 329-336). Morgan Kaufmann Publishers Inc. 
Koren, Y. (2008). Factorization meets the neighborhood: a multifaceted collaborative filtering model. In Proceedings of $K D D$ (pp. 426-434). ACM.

Koren, Y. (2010). Collaborative filtering with temporal dynamics. Communications of the ACM, 53(4), 89-97.

Koren, Y., \& Sill, J. (2011). OrdRec: an ordinal model for predicting personalized item rating distributions. In Proceedings of the 5th ACM conference on Recommender systems (pp. 117-124). ACM.

Koren, Y., Bell, R., \& Volinsky, C. (2009). Matrix factorization techniques for recommender systems. IEEE Computer, 42(8), 30-37.

Liu, N. N., Zhao, M., \& Yang, Q. (2009). Probabilistic latent preference analysis for collaborative filtering. In: Proceedings of CIKM (pp. 759-766). ACM.

Liu, S., Tran, T., Li, G., \& Jiang, Y. (2014). Ordinal random fields for recommender systems. In Proceedings of the 6th Asian conference on machine learning, JMLR: workshop and conference proceedings (pp. 283-298).

Ma, H., Zhou, D., Liu, C., Lyu, M. R., \& King, I. (2011). Recommender systems with social regularization. In Proceedings of the 4th ACM international conference on Web search and data mining (pp. 287-296). ACM.

McCullagh, P. (1980). Regression models for ordinal data. Journal of the Royal Statistical Society, Series B, 42(2), 109-142.

McFadden, D. (1980). Econometric models for probabilistic choice among products. Journal of Business, 53(3), S13-S29.

McNee, S.M., Riedl, J., \& Konstan, J. A. (2006). Being accurate is not enough: How accuracy metrics have hurt recommender systems. In CHI'O6 extended abstracts on Human factors in computing systems (pp. 1097-1101). ACM.

Mohri, M., Rostamizadeh, A., \& Talwalkar, A. (2012). Foundations of machine learning. Cambridge: MIT Press.

Rendle, S., Freudenthaler, C., Gantner, Z., \& Schmidt-Thieme, L. (2009). BPR: Bayesian personalized ranking from implicit feedback. In Proceedings of UAI (pp. 452-461). AUAI Press.

Resnick, P., Iacovou, N., Suchak, M., Bergstrom, P., \& Riedl, J. (1994). An open architecture for collaborative filtering of netnews. In Proceedings of the ACM conference on computer supported cooperative work (pp. 175-186). ACM.

Sarwar, B., Karypis, G., Konstan, J., \& Riedl, J. (2001). Item-based collaborative filtering recommendation algorithms. In Proceedings of the 10th international conference on World Wide Web (pp. 285-295). ACM.

Schacter, D. L., \& Dodson, C. S. (2001). Misattribution, false recognition and the sins of memory. Philosophical Transactions of the Royal Society B: Biological Sciences, 356(1413), 1385-1393.

Schein, A. I., Popescul, A., Ungar, L. H., \& Pennock, D. M. (2002). Methods and metrics for cold-start recommendations. In Proceedings of SIGIR (pp. 253-260). ACM.

Shi, Y., Larson, M., \& Hanjalic, A. (2010). List-wise learning to rank with matrix factorization for collaborative filtering. In Proceedings of the 4th ACM conference on Recommender systems (pp. 269-272). ACM.

Tran, T., Phung, D. Q., \& Venkatesh, S. (2007). Preference networks: Probabilistic models for recommendation systems. In Proceedings of the 6th Australasian data mining conference (pp. 195-202). ACS.

Tran, T., Phung, D. Q., \& Venkatesh, S. (2009). Ordinal Boltzmann machines for collaborative filtering. In Proceedings of UAI (pp. 48-556). AUAI Press.

Tran, T., Phung, D. Q., \& Venkatesh, S. (2012). Learning from ordered sets and applications in collaborative ranking. In Proceedings of the 4th Asian conference on machine learning, JMLR: Workshop and conference proceedings (pp. 427-442).

Weimer, M., Karatzoglou, A., Le, Q. V., \& Smola, A. (2007). Maximum margin matrix factorization for collaborative ranking. In NIPS (pp. 1593-1600). 